\title{
Editorial
}

\section{Central Nuclear Pharmacy, Our lost Treasure}

\author{
Bayomy, TB, PhD
}

Nuclear Medicine Department, King Hamad University Hospital, Kingdom of Bahrain

Commercial central radiopharmacy is a mature and practical concept in the developed countries long time ago. However, in the developing ones where its importance and feasibility is much more valid, this concept is not yet well established.

Since the running cost of nuclear medicine procedures is being considered the main obstacle of the NM progress in any developing country, it is clear that the central radiopharmacy can lead the NM progress and market in these countries ${ }^{(1)}$.

\section{What is the central radiopharmacy?}

It is a highly specialized nuclear pharmacy which is able to produce and distribute calibrated, GMP complied and safe single radiopharmacetical doses to the NM facilities in its geographical area in a routine and scheduled timetables.

A centralized nuclear pharmacy in any region having reasonable transportation facilities can lead to significant savings of money and personnel time. It must be shared by many, if not all, hospitals in a given region; otherwise it may not be feasible to run it economically.

The scope of the central radiopharmacy
Services can vary according to the capacity and needs of the served institutes. It can be assigned for PET and SPECT radiotracers or to only one of theses categories.

\section{What can a central radiopharmacy add to our nuclear medicine community?}

\section{Cost Effectiveness}

It is well known to the NM practitioners that the main challenges in this field are not the initial investments on the machines, hot lab and radiation protection tools, but the running cost of radiopharmaceuticals and cold kits that are not usually complying with the number of patient procedures performed.

The loss of Tc-99m activities that varies between $50 \%$ in busy institutes and $85 \%$ in low profile ones increase the cost of the $\mathrm{NM}$ procedures that limits the utilization of the NM technology due to this high cost in addition to the irregular availability of the tracers (Moly Crisis) ${ }^{(2)}$.

With the central radiopharmacy the cost can be reduced by up to $70 \%$ which will reduced the overall cost of the procedure what will encourage the referring physician to rely on the NM scans for their patients' diagnosis. 


\section{Procedure standardization and QA}

Although there is a great demand now allover the world for applying the standard international safety rules for patients' injections, most of the NM facilities are not able to do the proper radiopharmacy QC tests due to the lacking of the instruments and trained professionals.

With its mandatory and basic configuration, the central radiopharmacy can easily provide high caliber, safe and up to standard patient doses which is fulfilling the international drug safety demands.

\section{$\underline{\text { Radiation Protection Aspects }}$}

The radiation protection issues out of labeling and handling the NM radiopharmaceuticals is also an active professional concern.

It is mandatory that acentral radiopharmacy service will reduce the NM professionals' exposure as well as decreasing the probability of contamination. This is being highly appreciated and encouraged by both the governing authority and the NM radiation workers.

\section{$\underline{\text { NM Performance Enhancement }}$}

The central radiopharmacy can decrease the number of workers directly involved the hot lab procedures that serves for a better diagnostic and patient care features of the NM facility.

This can also acts as an efficient key factor in overcoming the worldwide shortage of the NM qualified professionals ${ }^{(3)}$.

\section{$\underline{\text { Nuclear Medicine Development }}$}

Most of the private Hospitals and
Most of the private Hospitals and radiology centers' efforts to fulfill their services' demands through adding NM units in their facilities are being tackled with the relatively high running cost of the NM radiopharmaceuticals and cold kits which is not affordable due to the irregular flow of patients.

This concept forced these centers to either ignore the idea or to establish a NM unit while maintaining the financial drawbacks out of this to complete their services.

The central radiopharmacy is used to be the ultimate solution for these challenges as these centers can easily invest in a Gamma Camera and a small dispensing lab and then pay only for the radiopharmaceuticals needed. The central radiopharmacy will also open the field for having NM services dedicated centers which can depend on the pharmacy for the supply of diagnostic and therapeutic agents whenever needed.

\section{$\underline{\text { Lower Licensing Class }}$}

Usually, in many countries, NM facilities with no labeling and dispensing activities, can apply for a lower grade licenses due to its limited activities whenever benefited from the central radiopharmacy services.

For these centers, this lower licensing class means lower cost of shielding, radiation monitoring system and less number of dedicated professionals.

\section{$\underline{\text { Research and Education }}$}

The central radiopharmacy in an area can lead the training, research and educational plans in the field of nuclear pharmacy and 
isotope production through its professional team of radiopharmacists, radiochemists and technologists.

It can provide guidance and technical advices to the concerned facilities for properstandardization of theNM procedures and related dosimetryfeatures ${ }^{(4)}$.

\section{What is the Central Nuclear Pharmacy configuration?}

Since the centralized nuclear pharmacy serves a number of institutions in different locations, a precisely timed protocol is needed for procuring all the requisitions and then preparing and shipping radiopharmaceuticals to different customers at specified times.

Inner Unit Which is dedicated for the labeling and dispensing of the radiopharmaceutical doses?

\section{What is the Central Nuclear Pharmacy configuration?}

Inner Unit Which is dedicated for the labeling and dispensing of the radiopharmaceutical doses?

Outer Unit Contains proper transportation units which can maintain all radiation safety and GMP rules to deliver the doses to the concerned facilities

\section{$\underline{\text { Inner Unit }}$}

\section{a) Reception and administration area which is dedicated for:}

1. Coordination of the flow inside and outside the central radiopharmacy.

2. Documentation of the dispensed doses and work flow characters.
3. Accommodate the staff rooms and daily activities.

b) Hot lab where all preparations and dispensing procedures are performed aseptically under the strict GMP regulation.

${ }^{99 m}$ Tc-radiopharmaceutical labeling procedures as well as dispensing the ready received doses of SPECT and PET RPhs are to be performed in a clean environment that can be obtained through either a Clean Room or Laminar flow isolator

Clean room this is the standard choice for the lab functions, however, it is very sophisticated and costly as it contains a high negative pressure air-lock system, laminar flow, preparation enclosure and dispensing unit.

Laminar flow isolator it is a practical and cost-effective alternative of the clean room solution. It has all features with a small footprint and with no special room arrangements.

a) Quality Control Lab To ensure that the highest quality standards are applicable for the dispatched doses. Most of the tests are pre-release ones; however, some of them are post-release.

b) We need a dedicated Quality Control Lab to perform the quality control procedures in a lower grade (clean) area and to avoid the high scattered radiation from the main lab area.

c) Health Physics Lab including the radiation monitoring tools as well as personal and area radiation safety items. All licensing, $\mathrm{QC}$, radiation survey and personal monitoring 
documents are recorded and organized in this area.

d) Radioactive waste storage room that can be categorized to solid, gas and liquid waste areas in huge Central Radiopharmacy facilities.

Packing and Dispatching area where the dispensed doses are being packed properly for the final shipment. This area should be close to a dedicated radioactivity exit facing the transportation vehicle parking area.

\section{$\underline{\text { Outer unit }}$}

Contains compact, lead lined and emergency equipped transportation vehicles that are able to reach close as well as distant facilities in a predefined time table in compliance with the radioactivity transportation regulations of the local governing authority ${ }^{(5)}$.

In master radiopharmacies where the services are being provided among a large number of cities and/or distant areas, the central radiopharmacy can act with public or own air transportation.

\section{Conclusion:}

The central radiopharmacy is being considered the lost treasure of nuclear medicine in our country. It is a practical and feasible solution to overcome the persisting NM tackles out of the high running cost of any NM facility. However, it needs a full sharing responsibility between the radiopharmacy, clinical facilities in the area and the governing authority for a successful integration of this practicing in our developing communities.

\section{References}

\section{1- Allegra L. De Pietro. Medication} management in nuclear pharmacy. Pharmacy purchasing \&products, March, Vol 9, n. 3 page $32 ; 2012$.

2- Callahan RJ. The role of commercial nuclear pharmacy in the future practice of nuclear medicine. Seminars in nuclear medicine, Apr; 26 (2): 85-90; 1996.

\section{3- Elliot AT, Hilditch TE, Murray T,} McNutty H. The design and construction of a central radiopharmacy. Nuclear Medicine Communication, Apr; 14 (4) : 328-34; 1993.

4- Saha, GB. Fundamentals of Nuclear Pharmacy, Sixth ed, Springer New York; 2010.

\section{5- De Arellano, R, Piera C, Pavia J, Setoain}

J.Experiences in setting up the first centralized radiopharmacy in Spain. Nuclear Medicine Communication, 20 (3): 279-285; 1999. 